\title{
Description of an anesthesia/perioperative medicine rotation for post graduate year one pharmacy residents
}

\begin{abstract}
Purpose: The purpose of this descriptive report is to outline the structure of our post-graduate year one (PGY-1) rotation in anesthesia/perioperative medicine and to outline how we meet the PGY-1 ASHP residency accreditation standards through this learning experience.

Summary: Sparse evidence exists to describe pharmacist training opportunities in anesthesia/perioperative medicine. A PGY-1 rotation in anesthesia/perioperative medicine can prepare a pharmacist to practice in this setting and meet the PGY-1 ASHP residency accreditation standards through several learning experiences including pharmacy practice/service, compliance, education/scholarship, clinical experiences and transitions of care.

Conclusion: The PGY-1 rotation in anesthesia/perioperative medicine prepares a pharmacist to practice and assume leadership for perioperative medication management in the perioperative setting. PGY-1 pharmacy residency programs should consider a rotation in this setting as an opportunity to meet the growing need for pharmacists who can advance pharmacy practice and take on leadership roles in the perioperative setting.
\end{abstract}

Keywords: pharmacy, ASHP accreditation standards, pgy-1 pharmacy residency
Volume 4 Issue 3 - 2016

\author{
Kevin Cowart, ' Aranjeet Sidhu, ${ }^{2}$ Michael \\ Willis ${ }^{3}$ \\ 'PGY-I Pharmacy Resident, Duke Regional Hospital, USA \\ ${ }^{2}$ Clinical Pharmacist, Perioperative Services, Duke Regional \\ Hospital, USA \\ ${ }^{3}$ Clinical Coordinator \& PGY-I Residency Program Director, \\ Duke Regional Hospital, USA
} Correspondence: Kevin Cowart, PGY-I Pharmacy Resident, USA, Tel 9194704188, Fax 9194707384,

Email kevin.cowart@duke.edu

Received: May 05, 2016 | Published: May 24, 2016
Abbreviations: SCIP, surgical care improvement project; PACU, post-anesthesia care unit; NPSGs, national patient safety goals; OR, operating room; TJC, the joint commission

\section{Introduction}

The role of the pharmacist in the perioperative setting and operating room (OR) has long been well described. ${ }^{1}$ The implementation of an OR pharmacy and provision of clinical pharmacy services has been shown to reduce inventory costs, reduce lost revenue, improve medication distribution, improve patient charging, improve intravenous compounding and labeling, and improve narcotic control and accountability. ${ }^{1,2}$ Further, pharmacists trained in the perioperative setting can reduce the incidence of adverse drug events, improve compliance with surgical care improvement project (SCIP) measures, mitigate drug diversion and serve as a drug information specialist to surgeons, nurses and health care professionals working in the surgical or post-anesthesia care unit (PACU) setting. A pharmacist trained to provide pharmaceutical services in the perioperative setting is also better prepared to provide surveillance of adverse drug events in this setting and participate on multi-disciplinary committee's to improve health care delivery.

Over the last 25years the number of OR pharmacies in hospitals has more than doubled, yet few learning experiences for pharmacy students and pharmacy residents exist to meet this growing demand. ${ }^{3}$ Most pharmacists who work in the perioperative setting or in an OR pharmacy have learned to do so from on the job training, since much of the knowledge needed to function effectively in the perioperative setting is not taught in the doctor of pharmacy curriculum. To our knowledge the only literature describing a pharmacy student experience in the OR setting comes from The American Society of Health-System Pharmacists (ASHP), and one paper outlining the establishment of a clinical pharmacy OR rotation for pharmacy students. ${ }^{4,5}$ We identified no literature describing a pharmacy resident learning experience in the OR. The purpose of this descriptive report is to outline the structure of our post-graduate year one (PGY-1) rotation in anesthesia/perioperative medicine and to outline how we meet the PGY-1 ASHP residency accreditation standards through this learning experience.

\section{Rotation setting}

The PGY-1pharmacy residency program at our institution is 12 months in duration and consists of training in many aspects of pharmacy practice including core rotations in internal medicine, critical care, emergency medicine, surgery, anesthesia/perioperative medicine, infectious diseases and two electives. Longitudinal experiences include anticoagulation, nutrition support, research, administration and a pharmacy practice service commitment. Our anesthesia/perioperative medicine rotation is a 4 week required rotation that is spent primarily in the OR pharmacy. Our institution is a 369-bed community hospital within a large health-system and has 15 operating rooms, 2 labor and delivery rooms, 2 cystoscopy suites, an ambulatory surgical care unit consisting of 16 beds and a 15 bed PACU. We also have 4 exam rooms for outpatient/pre-operative assessments as a part of our outpatient services. The OR pharmacy provides pharmaceutical care services 16hours per day Monday through Friday and is staffed by one clinical pharmacy specialist and two pharmacy technicians. We describe in Table 1 the activities that are required during the 4 week rotation experience. 


\section{Goal of the rotation experience}

The goal of the rotation is to allow the PGY-1 pharmacy resident to become an independent pharmacy practitioner in the OR environment and an integral member of the surgery team. At the conclusion of the rotation, the resident will have the skills and practice attitudes necessary to establish and advance pharmacy practice in the OR environment. During the rotation, the resident is responsible for ensuring safe and effective therapeutic management of all surgical and post-operative pain and emesis medications. The resident is also responsible for completing daily audits of controlled substance inventory management and handling administrative responsibilities associated with the OR pharmacy, such as authorizing access to medication distribution systems for OR personnel. Other pharmaceutical services provided by the resident may include quality improvement projects and formulary management.

\section{Evaluation strategy \& learning objectives}

Our PGY-1 pharmacy residency program utilizes Pharm Academic to evaluate and document achievement of the PGY-1 pharmacy residency objectives. A formative self-evaluation is completed by the resident prior to the start of the rotation, a midpoint evaluation is completed by the preceptor at the end of week 2 , and a summative evaluation is completed by the resident and preceptor at the conclusion of the 4 week rotation. We describe in Table 2 how we meet the goals and objectives for the PGY-1 pharmacy residency program with the anesthesia/perioperative medicine rotation. The preceptor serves to facilitate, coach and mentor the pharmacy resident throughout the learning experience. At the beginning of the rotation an orientation is provided and the preceptor orients and introduces the pharmacy resident to OR staff, as well as policies and procedures.

Table I Activities completed during the 4week anesthesia/perioperative medicine rotation

\begin{tabular}{l}
\hline Service component \\
\hline Dispensing of perioperative medications \\
Preparation of sterile products per USP 797 protocol \\
Medication order verification \\
Compliance measures \\
Ensure compliance measures with SCIP,TJC and the NPSGs \\
Participation in interdisciplinary meetings to develop hospital protocols \\
Narcotic diversion \\
Maintain controlled substance inventory \\
Training with pharmacy technician in narcotic dispensing, disposal and auditing \\
Education \& scholarship \\
New drug presentation \\
Topic discussions \\
Monograph preparation \\
Clinical experiences/transitions of care \\
Pre-operative clinic once weekly \\
Surgical procedure observation \\
Post-operative pain management rounds once weekly
\end{tabular}

SCIP, surgical care improvement project;TJC, the joint commission; NPSGs, national patient safety goals

Table 2 Taught and evaluated objectives for the 4week anesthesia/perioperative medicine rotation

Taught and evaluated objectives

Activities

Goal RI.I: In collaboration with the health care team, provide safe and effective patient care to a diverse range of patients, including those with multiple co-morbidities, high-risk medication regimens, and multiple medications following a consistent patient care process

Objective Interact effectively with health care teams

RI.I.I to manage patients' medication therapy
Provides prospective medication-regimen review and alerts providers of allergies, drug-drug interactions and adherence to appropriate institution practice guidelines. Rounds on post-operative anesthesia pain management team to provide safe and effective medication management. 
Table Continued..

\begin{tabular}{|c|c|c|}
\hline \multicolumn{2}{|c|}{ Taught and evaluated objectives } & Activities \\
\hline $\begin{array}{l}\text { Objective } \\
\text { RI.I.2 }\end{array}$ & $\begin{array}{l}\text { Interact effectively with patients, family } \\
\text { members, and caregivers }\end{array}$ & $\begin{array}{l}\text { Evaluate a patient's home medications at the preoperative anesthesia interv } \\
\text { and provide appropriate recommendations for perioperative medication } \\
\text { management. }\end{array}$ \\
\hline $\begin{array}{l}\text { Objective } \\
\text { RI.I.7 }\end{array}$ & $\begin{array}{l}\text { Document direct patient care activities } \\
\text { appropriately in the medical record or } \\
\text { where appropriate }\end{array}$ & $\begin{array}{l}\text { Enter allergies and pharmacotherapeutics interventions into the electronic } \\
\text { medical record (EPIC). }\end{array}$ \\
\hline
\end{tabular}

Goal RI.3: Prepare, dispense, and manage medications to support safe and effective drug therapy for patients

\begin{abstract}
Objective Prepare and dispense medications following
RI best practices and the organization's policies and procedures
\end{abstract}

Dispense medications by appropriately compounding, packaging and labeling pharmaceuticals. Prepare medications with sterile technique and intravenous admixture with special emphasis on USP 797 protocols.

Maintain an accurate daily controlled substance inventory.

Manage and understand policy and procedure regarding narcotic vault inventory including controlled medications. Provide medication order entry and verification of anesthetic and surgical medications including but not limited to epidural, TAP block and nerve block orders.

\section{Goal R2.I: Demonstrate ability to manage formulary and medication-use processes, as applicable to the organization}

Objective

R2.I.I

Prepare a drug class review, monograph, treatment guideline, or protocol

Objective

R2. I.2

Objective

R2.I.4
Participate in medication event reporting and monitoring
Prepare a new drug monograph for presentation at pharmacy \& therapeutics committee meeting.

Participate in interdisciplinary committee meetings for effective protocol development

Complete a medication use evaluation or cost-analysis of an anesthetic or surgical medication to evaluate its place in current practice.

Investigate, analyze and prepare medication adverse event reports and enter them into the health system reporting database. Monitor appropriateness of stock in anesthesia work stations and separation of look-alike-sound-alike medications. Apply safe medication practices to enhance patient safety.

Goal R4.I: Provide effective medication and practice-related education to patients, caregivers, health care professionals, students, and the public (individuals and groups)

Objective

R4.I.I

Design effective educational activities

Objective Use effective presentation and teaching skills

R4. I.2
Prepare daily/weekly topic discussions for surgical, pharmacy or other clinical staff.

Presentation of a formal in-service to the anesthesia department, either as a medication use evaluation or a case report through a morbidity and mortality discussion.

\section{Goal R4.2: Effectively employs appropriate preceptor roles when engaged in teaching students, pharmacy technicians or} fellow health care professionals

Objective Effectively employ preceptor roles, as

R4.2.2 appropriate

Precept and evaluate pharmacy students, where appropriate. 


\section{Discussion}

\section{Service component}

The PGY-1 resident is expected to provide preparation of sterile products, dispensing of perioperative medications and medication order verification. These activities will help the pharmacy resident in becoming proficient in medication management in the perioperative setting. We ensure that drug distribution and drug preparation are not so labor-intensive that it would exclude time for providing clinical pharmacy services. After the resident demonstrates proficiency in drug preparation and distribution, a large amount of these daily function are handled by OR pharmacy technicians. We also consider adverse-drug and event reporting as well as participation in emergency life support (ACLS/BLS) as part of the daily service component to function as an integral member of the surgical team.

\section{Compliance measures}

A pharmacist trained in perioperative setting has the potential to impact three SCIP compliance measures including achieving sufficient antibiotic levels, appropriate antibiotic selection, and preventing antimicrobial-resistant pathogens. A PGY-1 pharmacy resident training in the perioperative environment has the potential to largely impact and measure compliance with these measures. ${ }^{6}$ Our learning experience involves the resident in interdisciplinary meetings regarding medication safety and perioperative services to directly learn how to develop effective protocols and meet compliance measures outlined in the SCIP guidelines, The Joint Commission (TJC) medication management standards and the National Patient Safety Goals (NPSGs). Our PGY-1 pharmacy resident is also responsible for communicating with stakeholders across our health-system regarding these measures.

Narcotic diversion is an ongoing concern among all healthcare professions. Pharmacists can play a large role in the management and reduction of narcotic diversion in the perioperative setting. ASHP has also recognized the growing concern for diversion in the OR setting and recommends that a OR pharmacist actively participate in the dispensing, disposal and audit of controlled substances in the perioperative setting. ${ }^{7}$ The PGY-1 pharmacy resident gains handson experience in the narcotic diversion process through completing two days of training with an experienced pharmacy technician in the dispensing and disposal of controlled substances in the OR. After completing this training, the resident is responsible for identifying potential flaws and areas for improvement in our medication-use and distribution process in the OR. The resident is also responsible for completing and maintaining daily audits of controlled substances and post-case-reconciliation reports in the OR.

\section{Education \& scholarship}

One of the many roles of the pharmacist is to educate staff about medication and hospital policy regarding the medication use process. Unfamiliarity with medication management policies in the perioperative setting is common and a pharmacy resident can effectively educate nursing and medical staff in the perioperative environment to ensure compliance with medication use. Our resident is required to deliver at least one in-service to nurse anesthetists and deliver one formal presentation on a new drug or recent formulary approval. This presentation is delivered to anesthesia staff at a weekly department meeting. Writing skills are enhanced through the development and completion of a new drug monograph assignment which is brought forward to the pharmacy and therapeutics committee or health-system formulary approval. The resident is also responsible for handling any drug information requests or cost-utilization analysis requests that arise throughout the rotation experience. To improve teaching skills, the resident prepares a formal topic discussion on the topics outlined in Table 3. Pharmacists, current pharmacy students on rotation at the hospital and other surgical staff are invited to participate in these topic discussions. Additional topics may be assigned or topics changed based on interests and needs from the department or hospital. The resident is also required to enhance their precepting skills by serving as a co-preceptor for introductory and advanced pharmacy rotation students if they are on rotation simultaneously.

Table 3 Resident-led topic discussions during the 4week anesthesia/ perioperative medicine rotation

Inhaled, local \& intravenous anesthetics

Benzodiazepines, opioid agonists/antagonists \& non-opioids

Neuromuscular blocking agents \& reversal agents

Perioperative anticoagulation

Post-operative pain \& emesis

Anti-cholinesterase drugs and cholinergic agonists

\section{Clinical experience \& transitions of care}

To enhance the resident's clinical learning, we have the resident observe a number of common surgical procedures during the rotation with the primary goal of gaining a better understanding of the medications utilized in different surgeries. Table 4 describes the typical surgeries and instrumentation that the resident will observe during the 4 week learning experience. The resident is asked at the beginning of the rotation which surgeries they have previously observed and if there is any specific interests or procedures that the resident would like to observe.

In an effort to expose the resident to the full care transition for surgery patients, we have the resident spend one half-day per week in the pre-operative clinic completing medication reconciliation and advising patients on proper perioperative medication management. The resident works in this setting collaboratively with nursing and medical providers and is responsible for identifying new areas for pharmacist involvement in this setting and how to improve the care transitions for patients in our institution. In addition to time spent in the pre-operative clinic, our resident spends time rounding one morning per week with the post-anesthesia pain management care team. The PGY-1 pharmacy resident is responsible for identifying and recommending optimal pain management modalities for post-surgical patients on this care team. The resident documents all interventions in an effort to build practice habits that demonstrate accountability and the value for clinical pharmacy services.

\section{Challenges, limitations \& opportunities}

According to Holm and Brinkman, one of the greatest challenges to developing a pharmacy student OR rotation was the lack of time that a pharmacist can dedicate each day to teaching in the OR setting. ${ }^{5}$ Many times, the pharmacist is the sole provider for pharmaceutical care services in this setting, and may not have the opportunity to dedicate an appreciable amount of time towards mentoring and teaching 
pharmacy students. This limitation from a supervisory standpoint can be overcome with a PGY-1 pharmacy resident in this setting, since a PGY-1 pharmacy resident is a licensed pharmacist who can perform order verification and function as an independent pharmacist.

Table 4 Examples of surgeries, procedures and instrumentation observed during the 4 week anesthesia/perioperative medicine rotation

\begin{tabular}{ll}
\hline Surgeries/procedures & Instrumentation \\
\hline $\begin{array}{l}\text { Epidural and spinal injections } \\
\text { Peripheral nerve block } \\
\text { placement }\end{array}$ & Anesthetic gas machine \\
$\begin{array}{l}\text { Intubation and airway } \\
\text { management }\end{array}$ & Cell saver \\
$\begin{array}{l}\text { Coronary Artery Bypass Graft } \\
\text { (CABG) }\end{array}$ & Bispectral index monitor \\
$\begin{array}{l}\text { Pacemaker placement and } \\
\text { cardio version }\end{array}$ & \\
Cardiac catheterization &
\end{tabular}

MRI with anesthesia

Transversus abdominus plane (TAP) block observation

Knee \& hip surgeries

\section{Future direction}

Our future plans for this learning experience include providing more clinical rounding exposure on the pain management team. Our institution is in the process of developing a post-anesthesia pain management rounding team and the pharmacy resident involvement on this team would help ensure safe and effective pain management for post-operative patients. We are also exploring the feasibility and need for patient education and medication discharge counseling by a pharmacist for patients who received peripheral nerve blocks and other long-acting anesthetic agents.

Our institution also requires the PGY-1 pharmacy resident to complete a 4week surgery rotation on a general and bariatric surgery floor. We are exploring how we can meet the ASHP residency goal R1.2 of ensuring continuity of care during patient transitions between care settings. To fully immerse the PGY-1 resident in the care transitions for surgical patients, we would like to meet this objective by integrating the surgery and the anesthesia/perioperative medicine rotations. We are exploring if cross-covering order verification or participating in multiple patient-care rounds in the PACU and surgery floors would be the most effective approach.

\section{Conclusion}

Our ASHP-Accredited PGY-1 pharmacy residency includes a required 4 week anesthesia/perioperative medicine rotation. We have continued to develop the rotation based on current resident feedback in conjunction with the identification of innovative practice models for pharmacists in the OR setting. We are fortunate to have a strong relationship with our medical and nursing departments that foster interdisciplinary patient care, and largely attribute the success of our anesthesia/perioperative medicine rotation to these relationships. The ASHP Guidelines on Surgery and Anesthesiology Pharmaceutical Services provide a good overview of the role of a pharmacist in the OR setting. ${ }^{7}$ To continue to develop and fill positions in the OR setting, pharmacy residency programs should consider requiring a rotation in this setting as an opportunity to meet the growing need for pharmacists who can advance pharmacy practice and take on leadership roles in the perioperative setting.

\section{Acknowledgements}

None.

\section{Conflict of interest}

Author declares that there is no conflict of interest.

\section{References}

1. Swinney DC, Anthony J. How were new medicines discovered? Nat Rev Drug Discov. 2011;10(7):507-519.

2. Takenaka T. Classical vs reverse pharmacology in drug discovery. $B J U$ Int. 2001;88 (Suppl 2):7-10.

3. Lazo JS. Rear-view mirrors and crystal balls: a brief reflection on drug discovery. Mol Interv. 2008;8(2):60-63.

4. Surh YJn. Reverse pharmacology applicable for botanical drug development - inspiration from the legacy of traditional wisdom. J Tradit Complement Med. 2011;1(1):5-7.

5. Patwardhan B. Reverse Pharmacology and Systems Approaches for Drug Discovery and Development. Current Bioactive Compounds. 2008;4(4):201-212.

6. Guantai E, Chibale K. How can natural products serve as a viable source of lead compounds for the development of new/novel anti-malarials? Malar J. 2011;10(Suppl 1):S2.

7. Vaidya ADB. Reverse pharmacology-a paradigm shift for drug discovery and development. Curr Res Drug Discover. 2014;1:39-44. 